\title{
La crisis del Atlántico Sur y su influencia en el sistema regional*
}

El conflicto anglo-argentino del Atlántico Sur ha surgido en el marco de un proceso de cambio que venía ocurriendo en las relaciones internacionales dentro del hemisferio occidental, tanto en lo que se refiere a las vinculaciones entre los pafses de América Latina como a las modalidades de interacción entre estos últimos y los Estados Unidos. El funcionamiento del Sistema Interamericano y sus expectativas de eficacia, las categorías tradicionales que guiaban la inserción de América Latina en el Sistema Internacional o los procesos del Tercer Mundo, habían sido objeto de replanteamiento y nuevas definiciones como expresión de ese proceso de cambios, entre muchas otras materias.

A la luz de la existencia de ese conflicto y su desarrollo, ha sido planteada la interrogante de cómo incidirá esta situación en esa evolución. Para un punto de vista la cuestión ha sido enfocada como un problema meramente coyuntural, suponiendo que tan pronto concluya se regresará al estado anterior. Para otro punto de vista, en cambio, se producirán graves y permanentes consecuencias que alterarán esas relaciones de una manera muy profunda.

Este artículo tiene por objeto examinar el problema en función de las complejas características que ha tenido la evolución histórica de las relaciones interamericanas y regionales, perspectiva que permitirá identificar muchos aspectos que se encontraban presentes con anterioridad al actual conflicto. Sobre esta base se procurará distinguir entre los factores propiamente circunstanciales y aquellos que pueden tener un efecto permanente en el cuadro de las relaciones hemisféricas.

\section{Elementos negativos de una relación histórica}

Una primera consideración que se impone en el análisis de este problema es de carácter histórico. Las dificultades que puedan

* Versión preliminax de un artículo que ha sido sometido a una publicación en idioma inglés. 
afectar las relaciones entre América Latina y los Estados Unidos en el futuro, no son siempre una consecuencia principal del conflicto del Atlántico Sur sino de la naturaleza y evolución que ha tenido ese sistema de relaciones a lo largo de su historia. Ese conflicto representa un nuevo factor de complicación en un cuadro general, que en muchos casos constituye una circunstancia agravante de las tendencias que ya se venían manifestando.

Desde que las relaciones interamericanas comenzaron a intensificarse en la última parte del siglo pasado, ellas fueron presididas por ciertos síntomas negativos. El predominio de los Estados Unidos en la política regional fue seriamente resentido, sobre todo en los países del Cono Sur latinoamericano, que vieron en ese propósito el interés de rivalizar con sus propias aspiraciones en el ámbito sudamericano. Tanto Argentina como Chile fueron manifiestos opositores de la política hegemónica de los Estados Unidos, dando lugar a una diplomacia de confrontación. que habría de perdurar por mucho tiempo. En el caso argentino puede afirmarse que esa confrontación ha sido históricamente la regla general, exceptuándose breves períodos de acercamiento.

Un segundo carácter negativo del sistema, fue que él frustró las pérspectivas de una identificación regional latinoamericana, que se vieron diluidas en el contexto de un marco político interamericano. Las rivalidades y conflictos de poder entre los propios países de la región contribuyeron igualmente a facilitar esa frustración, a pesar de que este factor - que continúa presente- es rara vez admitido por los latinoamericanos.

El hecho concreto es que el Sistema Interamericano se consolidó y sirvió de manera importante a los intereses de los Estados Unidos hasta el término de la segunda guerra mundial. Sin embargo, los referidos factores de resentimiento, frustración y confrontación permanecerían subyacentes para emerger nuevamente en diferentes circunstancias históricas. Una de ellas, aunque no la única, ha sido precisamente la actual coyuntura del Atlántico Sur.

Junto con finalizar là segunda guerra mundial, Estados Unidos emprendió de manera decidida la construcción de una política exterior global. Pero en esa misma medida se inició un proceso de desvinculación de América Latina, región que pasó a ser una de muchas dentro de ese contexto global, lo que igualmente se tradujo en un creciente sentimiento de frustración entre los países latinoamericanos. Esta vez, sin embargo, la frustración tendría más bien un alcance económico que político, pues los países de la región aspiraban infructuosamente a beneficiarse de la prosperidad económica que habían alcanzado los Estados Unidos.

La pérdida de interés en los mecanismos del Sistema Interamericano y de la OEA puéde apreciarsè de manera muy evidente en la 
política seguida por los Estados Unidos durante ese período. El programa de la Alianza para el Progreso representö una excepción parcial en este proceso, pero a la vez estaba íntimamente vinculado a la política global de los Estados Unidos, siendo su origen inseparable de la aparición de la guerra fría y del fenómeno cubano en el continente americano. Con todo, su posterior fracaso llevaría no solamente a su abandono formal sino también a un nuevo aumento de los niveles de frustración de los países latinoamericanos.

La década de 1970 marcó una cierta reversión del ciclo globalizante de la política exterior de los Estados Unidos. La guerra de Vietnam, las crecientes dificultades económicas, el incremento del poderío estratégico de la Unión Soviética y otros factores, marcaron un límite al poder de los Estados Unidos en el mundo. A partir de entonces, junto con reconocerse este fenómeno, comienza a revalorizarse por los Estados Unidos la colaboración que pudieran prestar a sus objetivos de política exterior determinadas regiones o paises.

Este nuevo enfoque determinaría un resurgimiento relativo del interés por América Latina. Esfuerzos como el Nuevo Diálogo interamericano, que promovió Henry Kissinger desde la Secretaría de Estado, reflejaban este renovado interés, pero él no lograría materializarse, pues en cierto modo se trataba de una iniciativa tardía y ya la propia política exterior latinoamericana había cambiado de rumbo

Si bien la administración del Presidente Carter mantuvo este interés por América Latina, ello se tradujo en declaraciones más bien nominales que en un contenido efectivo. El ambiente de antagonismo y confrontación llegó quizás a su máxima expresión en las relaciones interamericanas. El distanciamiento que se produjo de Argentina y Chile por la situación de los Derechos Humanos fue muy grande, pero también el mismo fenómeno se dio en el caso de otros países por diferentes motivos, ya fueran éstos la política nuclear, los conflictos pesqueros $u$ otras causas. La aplicación de sanciones económicas, militares y políticas pasó a ser un hecho frecuente en el marco de estas relaciones. Independientemente del punto de vista que se tenga sobre su justificación, ello hacía imposible eI replanteamiento de las relaciones hemisféricas en un sentido positivo. El resultado inevitable de este distanciamiento y antagonismo fue el surgimiento de elementos de desconfianza entre los Estados Unidos y una buena parte de América Latina, con particular énfasis en los países del Cono Sur.

Este clima histórico y político negativo todavía perduraba en las relaciones hemisféricas al iniciarse el conflicto del Atlántico Sur, sin que las políticas de la administración Reagan hubieran logrado cambiarlo de manera significativa. 


\section{La búsqueda de la identidad regional y de la autonomia exterior}

En la misma medida en que Estados Unidos fue perdiendo su in. terés en América Latina y en el funcionamiento del Sistema Interamericano, las condiciones generales del Sistema Político Regional se fueron haciendo mạ́s permisibles. Esta circunstancia fue bien aprovechada por los palses latinoamericanos para iniciar la búsque. da de los dos objetivos que el Sistema Interamericano habia contribuido a frustrar: la identidad regional y el logro de la autonomfa política, particularmente en el plano exterior.

El primer objetivo se tradujo, desde comienzos de la década de 1960, en la organización y puesta en marcha de los diversos procesos de integración económica en la región. I.a Asociación Latino. americana de Libre Comercio, el Mercado Común Centroamericano, la Zona de Libre Comercio del Caribe, la Comunidad del Caribe o el Grupo Andino. fueron las expresiones más sobresalientes de esta inquietud. No por coincidencia fueron los paises del Cono Sur los más activos propulsores de este esquema, que permitfa retomar una línea histórica que habra resultado interrumpida. Sin embargo, después de diez años de esfuerzos sostenidos se llegaría a una nueva fase de frustración.

La segunda característica predominante del perfodo serfa la búsqueda de la autonomia en la conducción de las respectivas politicas exteriores. En este plano, se produjo una verdadera explosión en la actuación intertracional de América Latina. Ejemplos de lo anterior pueden encontrarse en el inicio de la polftica tercermun dista de México, que alcanzaría su expresión cúlmine bajo el gobierno del Presidente Echeverria; en las reivindicaciones económicas internacionales impulsadas por Venezuela, principalmente bajo el mandato de Carlos Andrés Pérez; en la polftica de independencia seguida por Brasil, una de cuyas expresiones importantes se dio en el campo nuclear; o en la determinación argentina de alcanzar el status de potencia, que fue bien resumido en el slogan de "Argentina potencia".

El fenómeno de la autonoma no se restringiria a los paises mayores de la región, sino que también se observa en el caso de las naciones intermedias y hasta en el de las pequeñas. El tercermundismo peruano; la apertura internacional de Chile, principalmente bajo los gobiernos de Alessandri y Frei; el despertar internacional de Colombia, con fuerte influencia en el seno de las organizaciones internacionales; y la propia presencia de Cuba en Africa, son todas expresiones de la misma tendencia, que adoptaba diferentes manifestaciones ideológicas o políticas según las caracteristicas de cada pais. Los nuevos tratados del Canal de Panama y la evolucion 
de las condiciones del 'Caribe, fueron también reflejo de esta realidad.

Si bien las causas que pudieron llevar a cada país a decidir su actuación u orientación internacional fueron ciertamente diferentes, es evidente que la región latinoamericana en su conjunto comenzó su rápida inserción en un sistema de política global. El desempeño de un papel propiamente internacional del Brasil, el ya mencionado liderazgo del Tercer Mundo a que aspiró México, o 'la búsqueda de Argentina en un papel como potencia occidental, son expresiones de este proceso de globalización política.

La globalización económica de las políticas latinoamericanas fue todavía más evidente, dándose lugar a un proceso de inserción y amplia participación en la economf́a mundial. Ya se tratara del comercio latinoamericano con los mercados europeos, soviético, japonés o chino, de la política seguida en relación a las materias primas, o de la presión internacional ejercida a través de mecanismos como el Grupo de Ios 77 o el Movimiento de los Países No Alineados, la orientación global era una característica evidente. Acciones regionales externas, como la política de Brasil en Africa o la de Chile en el Pacífico, corresponden también a este mịsmo esquema.

Dadas las orientaciones que habian seguido Estados Unidos y América Latina como los dos segmentos fundamentales del Sistema Interamericano, la crisis de la OEA y del sistema en su conjunto se hizo inevitable. Ello se tradujo primero en la difícil situación económica por la que han atravesado los organismos interamericanos $y$, en seguida, en la crisis política que habrá oportunidad de examịnar más adelante en relación al problema de las Malvinas. Tampoco el Banco Interamericano de Desarrollo y otros organismos regionales habrian de escapar a esta crisis.

Se creó así un importante vacío en las relaciones entre Estados Unidos y América Latina, que coincidió con las características de antagonismo que tuvieron esas relaciones bajo la administración del Presidente Carter. La iniciativa de crear el Sistema Económico Latinoamericano (SELA), fue originalmente concebida para aprovechar esa crisis en función de la creación de un organismo político y económico latinoamericano, que viniese a reemplazar a los mecanismos propios del Sistema Interamericano. Sin embargo, ella fue dotada de un fuerte componente ideológico por parte de los gobiernos de Pérez, en Venezuela, y Echeverría, en México, que fueron sus principales impulsores, orientación que no podia ser compartida por varios otros gobiernos de la región. SELA se transformó así en un mecanismo de acción económica de limitadas competencias.

Hacia mediados de la década pasada, América Latina se encontraba una vez más fuertemente dividida, retomando también en es- 
te punto sus más negativas tradiciones históricas. Los antagonismos ideológicos y el recrudecimiento de los conflictos fronterizos por doquier, acompañados de un clima de creciente hostilidad, desconfianza y armamentismo, habrían cle tener dramáticas consecuencias, algunas de las cuales no han sido ajenas a la crisis del Atlántico Sur. Sobre todo, este cuadro se traduciría en la frustración del objetivo integrador que se ha comentado, llevando, por consiguiente, a la imposibilidad de alcanzar establemente el propósito de la identidad latinoamericana que se había buscado.

La Asociación Latinoamericana de Libre Comercio fue gradua!mente privada de su dinamismo comercial inicial, para transformarse recientemente en un nuevo organismo de carácter muy débil. Es importante observar, a este respecto, que las iniciativas destinadas a reestructurar ALALG fueron planteadas por Argentina, país que buscaba adquirir una nueva libertad comercial, para así mantenerse vinculada o desvinculada de compromisos con la región, según lo dictasen las conveniencias de su estrategia exportadora global. El Mercado Común Centroamericano comenzó a desintegrarse a partir de la guerra entre El Salvador y Honduras en 1969 y llegaría a su colapso con la situación política interna que ha caracterizado a esa región durante los últimos años. Incluso los pragmáticos esquemas de la integración del Caribe entrarían en una etapa de serias dificultades. También el Grupo Andino, que en un momento era ejemplo de solidez, resultó afectado por la crisis general de la cooperación regional.

Es así como la década de 1970, a diferencia de la anterior, llegó a caracterizarse por una cooperación regional cada vez más débil, nominal y carente de contenido, dando lugar en su reemplazo a las profundas divisiones polfticas y económicas que se han señalado, así como a un creciente belicismo y tendencia al conflicto. Este negativo panorama aún permanece, habiéndose quizás agravado, lo que también es un hecho que debe tenerse presente para apreciar las implicaciones del actual conflicto del Atlántico Sur.

Así como en el ámbito interno de la región se frustraba el logro del objetivo integrador, la búsqueda de su dimensión global externa también encontraba dificultades de otra naturaleza. Manteniendo un cierto paralelismo con lo que había sido la experiencia de los Estados Unidos en la prosecución de su política global en su propia escala, también los pafses latinoamericanos comenzaron a encontrarse con los límites del poder en el ámbito internacional. $\mathrm{La}$ crisis energética, por ejemplo, limitó las perspectivas de la actuación internacional del Brasil, que sin dejar de tener intereses globales inició un retorno hacia América Latina bajo la hábil conducción del Canciller Guerreiro. El Pacto Amazónico o la coopera- 
ción argentino-brasileña en la Cuenca del Plata serian resultados importantes de este retorno a la región.

En el caso de México, la posición tercermundista que caracterizó al gobierno de Echeverría se demostró poco convincente como fundamento de su proyección internacional y fue reemplazada por una política más sólida bajo el gobiemo de López Portillo. Similares diferencias pueden apreciarse entre los gobiernos de Carlos Andrés Pérez y de Luis Herrera Gampins, en Venezuela, como entre el tercermundismo de los militares peruanos y el estilo sobrio de Belaúnde Terry. Chile encontró los límites de su apertura económica externa, sin perjuicio de los que tuvo en el plano politico; Colombia se vio afectada por un recrudecimiento de la violencia, mientras buena parte de Centroamérica se veía envuelta en la guerra civil. Cuba, dentro de su propia ideología, comenzó a sufrir las consecuencias de su política africana y Jamaica debió pasar a concentrarse en las necesidades de su desarrollo.

Quizás el proceso más difícil en este otro plano fue el de Argentina. Desde la muerte de Perón, Argentina inició un rumbo incierto que no habría de aclararse bajo sus sucesivos gobiernos militares. EI deseo de verse reconocida como una potencia occidental no podía prosperar, entre otras causas por la naturaleza de esos gobiernos. El tercermundismo fue siempre mirado con recelo en Buenos Aires, con lo cual tampoco se optó por esta alternativa. Su poIf́tica latinoamericana fue también conflictiva en algunos casos, sobre todo por su manejo del problema limitrofe austral con Chile. No obstante esta incertidumbre en su conducción política, a partir de la designación de Oscar Camilión en la Cancillería argentina, se observa también el deseo de fortalecer los vínculos con América Latina, fundamentándose para ello en su exitosa experiencia como embajador en Brasilia.

Por diferentes razones y experiencias, todos los países de la región, después de su búsqueda de la globalización externa, comienzan a revalorizar el espacio latinoamericano, buscando de una $u$ otra manera la reinserción en la política regional que anteriormente habfan abandonado, o por lo menos descuidado. El clima, de desintegración que se ha comentado harfa dificil este ejercicio, pero no por ello dejó de intentarse a través de diversas modalidades. Con la búsqueda de este reencuentro latinoamericano quedaba constituida la escena para los más recientes desarrollos.

\section{Ideologia y poder en la actual politica exterior interamericana}

Al asumir sus funciones el Presidente Reagan, comenzó el diseño de una política exterior que se basaba en valorizar el papel que debían desempeñar los países amigos de los Estados Unidos en la 
consecución de su nueva estrategia internacional. Esta última se inspiraba fuertemente en la necesidad de contrarrestar el poderío que entretanto había alcanzado la Unión Soviética y en el objetivo de superar las causas de la debilidad a que habían llegado los Estados Unidos, según ellas eran percibidas por la nueva administración. Los factores vinculados a una pugna ideológica y a una política del poder fueron explícitamente declarados como elementos centrales de la política exterior norteamericana.

Si bien la política latinoamericana de la nueva administración nunca llegó a definirse en términos demasiado explícitos, desde un comienzo pudo apreciarse que ella estaría inspirada en los mismos principios y factores que se han señalado. Ello parecía particularmente necesario frente al giro que comenzaban a tomar los acontecimientos en América Central, especialmente en relación a la creciente vinculación entre los regímenes de Nicaragua y Cuba y la explosiva situación de El Salvador.

En función de estos objetivos se procuró, en primer lugar, un acerçamiento con los países que más se habían alejado de los Estados Unidos bajo el gobierno de Carter, con particular referencia a los regímenes militares del Cono Sur. Por otra parte, se puso énfasis muy grande en la idea de establecer una relación especial con los países más poderosos de América Latina, donde destacaban Argentina, Brasil y México, así como parcialmente Venezuela. De esta manera, se reflejaba tanto la valorización de los factores ideológicos como los de poder en el marco de las relaciones con América Latina. Sin embargo, en esta misma medida se estaba limitando la efectividad de la política que se deseaba seguir.

Las señales de acercamiento ideológico fueron en cierto modo mal interpretadas en el Cono Sur, dándoseles quizás un alcance que iba más allá del que en realidad intentaban tener. En particular, se pudo pensar que el gobierno norteamericano estaba dispuesto a hacer caso omiso de ciertas orientaciones políticas nacionales, incluyendo las de carácter humanitario, en reciprocidad por el apoyo que algunos países pudieran dar a la estrategia general o regional de los Estados Unidos. Fue sólo después de adoptar muchas medidas inciertas y de experimentar varias reacciones adversas que el Departamento de Estado se vio en la necesidad de explicitar que ese acercamiento no significaba identificación total y que su política no pretendía abandonar el pensamiento democrático y humanitario de la sociedad norteamericana. Pero esta tardanza tendría un costo importante.

El resultado de estos equívocos fue que en algunas modalidades del pensamiento del extremo nacionalismo comenzaron a adquirir vigor en Argentina, ocupando importantes posiciones en el esquema de poder. Así como estas formas de pensamiento se expresaban 
con violencia en el plano interno, el proceso no tardaría en comunicarse hacia una dimensión internacional. Dándose las circunstancias apropiadas surgirían corrientes caracterizadas por el belicismo y la agresividad. El acelerado proceso armamentista y el hecho de que ya en 1978 se había amenazado a Chile con Ia guerra, eran indicadores de esta tendencia que alcanzaría su clímax en el conflicto del Atlántico Sur. La capacidad de persuasión en que siempre confió Estados Unidos para mantener la paz en la región, sería enteramente insuficiente frente a este cuadro ideológico.

El segundo principio en que la administración Reagan basó su aproximación inicial a la política latinoamericana, también se constituiría en un factor limitante de sus propósitos de acercamiento. La idea de establecer una relación preferente con Argentina, Brasil y México, como los países más grandes de la región, produjo dos tipos de inconvenientes. El primero se refiere a la naturaleza de esas relaciones y a la manera como ellas fueron percibidas por las respectivas contrapartes. El segundo inconveniente se refiere a las dificultades que ello suscitó respecto de los demás países Iatinoamericanos, excluidos de esa atención preferente.

$\mathrm{Si}$ bien los tres países escogidos miraban ciertamente con interés a los Estados Unidos, en su condición de potencia mundial y vecino hemisférico, ello no significaba que estaban dispuestos a realinear sus respectivas políticas exteriores, abandonando el ámbito de autonomía alcanzado luego de la evolución histórica que se ha indicado. En el caso de México, por ejemplo, se podía apreciar un interés en mantener la condición de uno de los principales socios comerciales de los Estados Unidos en el mundo, así como en desarrollar al máximo las reuniones consultivas de alto nivel -incluidas las visitas presidenciales y ministeriales-, pero manteniendo siempre sus propias orientaciones polfticas. La resistencia de México a verse asociado con Estados Unidos y Canadá en el marco de un esquema norteamericano, como el que postuló inicialmente la nueva administración de Reagan, o su línea de acción política en relación a Centroamérica, Cuba y el Caribe, son manifestaciones de esta última decisión.

La situación del Brasil era tambiên similar. El gobierno brasileño siempre ha apreciado sus vínculos con Estados Unidos y su status consultivo especial, pero tiene igualmente claros sus propios objetivos de política exterior, que no siempre son coincidentes con los del gobierno norteamericano. Podría incluso decirse que esos objetivos son cada día más discrepantes, pues el Brasil está empeñado en asegurar términos de negociación internacional consecuentes con sus propios intereses y realidades, fenómeno que todavía no pareciera haber sido bien comprendido en los círculos políticos y académicos de los Estados Unidos. 
El caso de Argentina es quizás el más notable en este plano. Precisamente porque Argentina no había logrado identificar todavía su papel internacional y no había tenido éxito en acreditarse como potencia occidental -entre otras causas por los factores ideológicos que se han indicado-, recibió con beneplácito el propósito de acercamiento del gobierno norteamericano, que le proporcionaba una oportunidad de rinculación con la mayor potencia de Occidente. Esta apertura se vio fuertemente reafirmada con la visita del General Galtieri a Washington en 1981, inmediatamente antes de asumir la presidencia de su país, con el distanciamiento de $\mathrm{Cu}$ ba y con la aceptáción del gobierno de Buenos Aires de colaborax con la política centroamericana de los Estados Unidos. La valorización del elemento ideológico y de poder que buscaba la política de Estados Unidos era precisamente lo que necesitaba el gobierno argentino, como una manera de reafirmar su incierto rumbo internacional.

Ello llevó a muchos analistas a asignarle a la Argentina el papel de aliado privilegiado de los Estados Unidos en América Latina. Efectivamente, todos los síntomas parecían asf indicarlo. Sin embargo, es interesante comprobar que nunca el gobierno de Buenos Aires pensó en abandonar la cosecución de sus propios objetivos de polf́tica exterior. Asf, por ejemplo, se continuó asignando especial importancia a la relación comercial que se habia establecido con la Unión Soviética a raf́ del embargo de granos y cereales decretado por algunos países occidentales, relación que fue ampliándose hacia otras esferas. La política nuclear, la ingeniería hidroeléctrica y el inicio de formas de cooperación militar han sido mencionadas como áreas posibles de entendimiento.

Más inquietante todavía fue la explicitación de algunos objetivos geopolíticos por autorizados voceros del nacionalismo argentino, que llegarían a ocupar altos cargos de gobierno. Se concebía para la Argentina un papel protagónico en la defensa de Occidente en el Atiántico Sur, frente a una supuesta amenaza soviética que podiría afectar a esta región. Si bien el Atlántico Sur se definía vagamente para este efecto, podía comprenderse pcrfectamente que lo que se buscaba era justificar la reclamación sobre las islas británicas, por una parte, $y$, por otra parte, aumentar la presión de las pretensiones argentinas sobre las islas chilenas situadas al sur deI Canal Beagle, controversia que se encontraba sometida a la mediación del Papa Juan Pablo Ir después que se logró evitar la guerra en 1978.

De esta manera, la Argentina mantenía la esencia de su tradición histórica, aún en la medida en que se registraba un proceso de convergencia con los Estados Unidos, el cual, según podría apreciarse después, habría servido más para justificar las aspiraciones argen- 
tinas que aquellas del gobierno de Washington. Todo ello tendría también una influencia directa en el desencadenamiento de la crisis del Atlántico Sur.

Este enfoque selectivo de la política de Estados Unidos hacia América Latina producirła también consecuencias de otro tipo en la región. De alguna forma parecía introducir la distinción entre paises de primera y segunda clase, lo que es muy ajeno a las tradiciones políticas latinoamericanas $y$, por ende, sumamente resistido Por esta causa, varios países que posiblemente hubieran mirado con interés el acercamiento de Estados Unidos a la región, se mantuvieron en cierta medida a la exceptativa, esperando que la política latinoamericana se definiera más precisamente y en función de ello poder especificar qué papel les cabría. En todo caso, no hubo una contribución positiva de este grupo, cuya influencia general era significativa.

La intención de acercamiento que tuvo el nuevo gobierno de los Estados Unidos no alcanzó la intensidad ni la efectividad que probablemente deseaban sus autores, debido a los problemas ideológicos y de poder que se han mencionado. Transcurrido el primer año de la administración Reagan, el enfoque más pragmático sustentado por el Secretario de Estado Alexander Haig comenzó a imponerse por sobre el de visiones ideologizadas en exceso, marcando en algunos casos una nueva orientación.

A pesar de ello, la mayor parte de los países latinoamericanos prefirieron mantener su compás de espera. Muchos de ellos habrían posiblemente deseado establecer una nueva relación de trabajo con los Estados Unidos, pero las condiciones no estaban dadas ni el ambiente tampoco invitaba a nuevos pasos en este sentido. De esta manera, los propósitos de acercamiento que pudiera tener una y otra parte de la relación interamericana no se materializaron en ningún sentido importante dada la incertidumbre existente en el significado de la política latinoamericana de los Estados Unidos y también debido a los temores de varios países de la región de verse instrumentalizados en función de problemas como la situación centroamericana, que ocupaba por cierto la primera prioridad del gobierno norteamericano y cuyas políticas no han sido en general compartidas en América Latina.

\section{Incidencia del medio internacional en el desencadenamiento de la crisis}

La decisión del gobierno de Buenos Aires de utilizar la fuerza para la recuperación de las islas británicas en el Atlántico Sur, vendria a precipitar los acontecimientos en las relaciones regionales e interamericanas. Si bien no es el propósito de este artículo anali- 
zar la crisis del Atlántico Sur como tal, se hace necesario mencionar algunos de sus elementos principales para apreciar la incidencia que el fenómeno podría tener en el futuro de las referidas relaciones.

En primer término debe retenerse el hecho de que esa decisión no fue enteramente independiente del curso que habla adoptado la política interna argentina. La creciente resistencia de la opinión pública a las políticas del gobierno militar, se había ya traducido en un nivel importante de confrontación, debiendo recordarse los serios incidentes que tuvieron lugar en Buenos Aires durante los días inmediatamente anteriores al inicio de las operaciones bélicas. Por otra parte, la presión de los grupos ultranacionalistas continuaba haciéndose presente en las alternativas de la política exterior argentina.

Paralelamente a las coyunturas internas, la escena internacional también parecía propicia para una decisión de esta naturaleza. Las relaciones entre Argentina y los Estados Unidos exan promisorias, hasta tal punto que se adelantaba un acuerdo entre ambos países para cooperar en el manejo de la explosiva situación centroamericana. La común preocupación geopolítica expresada por ambos gobiernos acerca del peligro del expansionismo soviético en el mundo, parecía avalar la posición argentina de constituirse en el guardián occidental del Atlántico Sur. De esta manera, la inserción de Argentina dentro del sistema occidental, que era la opción preferida por el gobierno de Buenos Aires, encontraba una justificación dentro de la política internacional general, contando con el aparente apoyo decidido de la primera potencia del mundo.

Las relaciones con Chile pasaban por un período de tranquilidad relativa debido a las tareas de la mediación pontificia, a pesar de lo cual se habían registrado numerosos incidentes en el extremo austral por violaciones de la soberanía marítima y aérea de Chile. De acuerdo a algunas versiones, ello también tenía relación con los planes de expansión geopolítica que se han señalado. Respecto de los demás países latinoamericanos, las relaciones eran normales y siempre cabía confiar en alguna forma de solidaridad.

Otros factores internacionales también parecían coincidir favorablemente. El Reino Unido no se mostraba particularmente inquieto por el futuro de las islas australes y se encontraba en una politica de reducción de su flota de guerra, con lo cual no cabía presumir una reacción tan fuerte como la que en definitiva tuvo lugar. Las relaciones con la Unión Soviética se desarrollaban también normalmente.

Gon todo, las reacciones que esa decisión suscitó fueron enteramente diferentes a lo que se habfa esperado. Si bien en el plano interno se generó una importante euforia nacionalista; que llevó a 
renovados niveles de popularidad al gobierno del General Galtieri, ello demostraría ser más bien transitorio y coyuntural. La solidaridad latinoamericana en que se confió, fue más bien calificada y sujeta a exigencias de importancia. El funcionamiento de los mecanismos interamericanos fue fundamentalmente ineficaz, como consecuencia de las diferentes percepciones de los países miembros y de otras razones que se comentarán más adelante.

El esquema de las relaciones con Estados Unidos pasó a una rápida situación de enfriamiento, y después de un período en que el Secretario de Estado procuró ejercer sus buenos oficios para alcanzar una solución pacífica se llegó a una política de nueva confrontación. La reacción del Reino Unido fue decidida y categórica. La actitud inicial de la Unión Soviética tampoco fue enteramente favorable a la causa argentina. El recurso a mecanismos como los no alineados o la solidaridad anticolonialista de algunos sectores del Tercer Mundo, resultarían también poco eficaces.

Este violento contraste entre expectativas y realidades, así como la puesta en movimiento de tantos y tan complejos factores de la política internacional, proporcionan las claves necesarias para poder analizar las posibles repercusiones que todo este proceso pudiera tener en los diferentes planos, sin dejar de tener en cuenta las circunstancias que derivan de la evolución histórica y de las políticas contemporáneas que se han venido comentando.

\section{El replanteamientio politico argentino}

Un primer elemento de cambio que debe tenerse presente es el relativo a la situación política interna de la Argentina. El agotamiento del esquema militar argentino venía produciéndose desde hacía ya muchos meses, generando la creciente resistencia politica que se señaló anteriormente, lo que unido a la crisis económica y otros factores determinó una situación difícilmente sostenible. Este proceso ciertamente se vio acentuado por la crisis del Atlántico Sur, que agravó Ios hechos políticos y económicos que lo habłan originado.

La fórmula política que había venido surgiendo con más vigor era la de un gobierno de transición civil sustentado por la Organización Multipartidaria, en que se combinan las fuerzas políticas tradicionales de la Argentina. La promesa del General Galtieri de proceder a la apertura democrática y a la dictación del estatuto de los partidos políticos envolvía dos pasos esenciales para que esa fórmula pudiera materializarse. Sin embargo, existían también dos problemas serios que superar. El primero era la carencia de liderazgo en el marco de los partidos políticos, que desde el fallecimiento de Perón y de Ricardo Balbin habían quedadio desprovistos de dirigentes poderosos. El segundo problema consistía en la natural 
resistencia que esta expectativa producía en el seno de las fuerzas armadas.

La crisis del Atlántico Sur vino a cambiar todo este cuadro de una manera dramática. La caída del gobierno del General Galtieri, el retiro de los Comandantes en Jefe de la Fuerza Aérea argentina y de la Armada, la separación de estas últimas dos ramas del gobierno y la asunción a la presidencia del General Reynaldo Bignone, determinarian un acelerado proceso de apertura política. Aun cuando éste no ha estado exento de riesgos, dada la resistencia de algunos sectores militares, ha ido hasta ahora progresando adecuadamente.

En cualquier alternativa política que surja de esta transición, cabe esperar también un cambio de importancia en la política exterior argentina, posiblemente en el sentido de regresar a la "tercera posición", que siempre caracterizó al peronismo, buscando la debida distancia de las principales potencias.. El distanciamiento que ya ha. tenido lugar de Estados Unidos y Europa Occidente, así como la prudente distancia que se mantiene de la Unión Soviética, harian que esta reorientación fuera la más natural. A la vez, ello significaría un renovado interés en el espacio político latinoamericano. La posibilidad de que la política argentina evolucionara hacia esquemas de izquierda es muy remota, pues ello es enteramente ajeno a la tradición del país y al sentir de las fuerzas armadas y de los partidos polfticos que hoy intervienen en el proceso de redefinición.

Dado el hecho de que la apertura política y la redemocratización argentina son uno de los resultados de la crisis del Atlántico Sur, los Estados Unidos se encuentran ante una situación paradojal. El acercamiento que se intentó con un régimen militar supuestamente afín, para asegurar la estabilidad del Atlántico Sur, el orden político interno y la paz en el Cono Sur, se vio enteramente frustrado al traducirse exactamente en los resultados opuestos. En cambio, el distanciamiento que probablemente intervendría con un, nuevo tipo de régimen político, sobre todo en la alternativa peronista, llevaría a los objetivos de estabilidad, orden y paz en que se fundamentaría su acceso al poder. Pocas dudas parecieran caber de que estos últimos son los factores que deben merecer la prioridad en América Latina. La reorientación política argentina, interna o externa, no puede considerarse tanto como una consecuencia de la crisis del Atlántico Sur -aun cuando ha sido por cierto influida por ella-, sino más bien como una parte integral de un proceso evolutivo que cuenta con su propia dinámica histórica.

\section{La redefinición latinoamericana}

El replanteamiento de las relaciones entre los propios países latino- 
americanos es un problema ya más complejo. A este respecto cabe recordar que América Latina, a la luz de la evolución histórica que se ha indicado anteriormente, se encontraba en una posición en cierto modo contradictoria. Por una parte, la política de rivalidad y cuasi confrontación se había acentuado seriamente durante los últimos diez años, hasta el punto de haberse traducido en factores de desintegración e inestabilidad, particularmente en el Gono Sur. Pero, por otra parte, la revalorización del èspacio latinoamericano representa también un fenómeno real que derivaba del retorno hacia la región después del periodo en que se buscaron las orientaciones globales que se han explicado. Esta última dimensión exigía el esfuerzo de generar nuevas formas de cooperación regional.

La crisis del Atlántico Sur intervino cuando esta dicotomía se encontraba en pleno funcionamiento, lo que quizá permite explicar lia actitud cautelosa observada por muchos de los países de la regiön. Si se pudiera identificar un común denominador, podría señalarse que si bien todos los paises de la región respaldaban la reclamación histórica de Argentina sobre las Malvinas, lo cual ha sido manifestado en más de una oportunidad, no compartían el uso de la fuerza para alcanzar ese objetivo, prefiriendo una solución negociada y resuelta pacíficamente. En parte ello respondía a principios que los palses latinoamericanos han reafirmado en su poiftica exterior general, pero también en parte a la inquietud de que el uso de la fuerza pudiera generalizarse en el continente americano para el efecto de respaldar las muchas cuestiones históricas que todavía se encuentran latentes.

Sobre la base de estas consideraciones es que Chile decidió seguir una política de neutralidad en relación al conflicto, en virtud de la cual no apoyaría a Argentina, pero tampoco haría nada que pudiera perjudicarla. Con todo, el hecho de que Chile fuese un país que no había valorizado el espacio latinoamericano en los mismos términos que los demás, unido al aislamiento en que se había ido colocando, contribuyó a crear una situación de cierta marginalidad respecto del resto de América Latina, to que, como se vèrá, es susceptible de provocar dificultades futuras.

En el caso de otros países, la expresión de esta dicotomfa fue más sutil. México expresó su solidaridad nominal con Argentina, pero se mantuvo enteramente al margen. Brasil, que ya se encontraba participando en un amplio proceso de cooperación con el gobierno de Buenos Aires, hubo de mostrar una mayor comprensión hacia la causa argentina, pero sin dejar de insistir en ningún momento en la necesidad de la solución pacífica del conflicto, respecto de lo cual emprendió varias pero infructuosas iniciativas diplomáticas. También Colombia siguió a este respecto una política cautelosa, expresiva de la solidaridad histórica general, pero fuerte- 
mente opuesta al uso de la fuerza en materia de conflictos territoriales. Los conflictos latentes con Venezuela y con Nicaragua hacían de esta posición una necesidad imperiosa para Colombia. Ecuador y Paraguay, con modalidades diferentes, mantuvieron asimismo una prudente distancia.

La regla general que se ha expuesto tendría, por cierto, algunas excepciones importantes. Venezuela fue, sin duda, el país de la región que comprometió más inequívocamente su apoyo a la reivindicación argentina, principalmente en virtud de los vínculos de entendimiento que siempre han unido a ambos países. Panamá observó una actitud similar pero menos influyente, posiblemente fundamentada en la cuestión de los tratados relativos al canal, como también lo hizo Bolivia, cuya dependencia de las fuerzas armadas argentinas es un hecho conocido, sin perjuicio del interés que tenía en revivir la cuestión de las reclamaciones en contra de Chile.

Perú representó un caso en cierto modo más difícil, pues, por una parte, aspiraba a solidarizar decididamente con Argentina, a la luz del entendimiento histórico y de la cooperación que existe entre los dos países, pero, por otra parte, tenía que observar una conducta compatible con la tradición pacífica de la diplomacia moderna del Perú, uno de cuyos distinguidos exponentes es el actual Secretario General de las Naciones Unidas. Es así como el sector castrense, representado por el Ministro de la Guerra, Luis Cisneros, impulsó la política de ayuda a la Argentina, en tanto que el sector civil y diplomático - encabezado por el Presidente Belaúnde Terry y su Canciller- pusieron especial énfasis en la solución pacífica del conflicto y emprendieron varias, pero también frustradas, gestiones de paz.

Las naciones del antiguo Caribe británico adoptaron una posición consecuente con su condición de miembros de la Comunidad Británica de Naciones, lo que tendría influencia en el contexto de la diplomacia multilateral.

A pesar de todas las diferencias, cautelas y salvaguardias que pudiesen existir, la diplomacia argentina actuó con agilidad en el plano latinoamericano, consiguiendo dos objetivos básicos. EI primero fue un grado importante de cooperación política, o al menos de neutralización, con Io cual aseguró que no tendría dificultades en su propio ámbito regional. Aun cuando no siempre consiguió Ia plenitud del apoyo buscado, obtuvo grados razonables de seguridad. El segundo objetivo, que también está vinculado al anterior, es que promovió la reactivación de algunas formas de vinculación económica, las cuales eran valiosas más que por su significado económico por su simbolismo político. Medidas adoptadas por el SELA y el Grupo Andino responden a este otro ángulo.

En lo coyuntural podría decirse que el escenario político latino- 
americano no cambió fundamentalmente como consecuencia del conflicto anglo-argentino, manteniendo la dicotomía entre confrontación y cooperación que ha sido característica del pasado, aun cuando no deben excluirse muchos problemas puntuales que se han presentado. Sin embargo, muy diferentes parecieran ser las tendencias que se insinúan hacia el futuro.. En este otro plano se ha hecho patente la necesidad de replantear muchos conceptos, políticas y orientaciones, las que probablemente habrán de traducirse en algunos cambios profundos y permanentes. Si bien varios de ellos están vinculados a problemas de la política interämericana, que se mencionarán más adelante, dos ejemplos pueden servir de ilustración.

El primero se refiere al caso del Brasil, país cuya reinserción en el ámbito latinoamericano labía sido más bien tenue. La principal preocupación del Brasil en materia de seguridad se referla a posibles hipótesị de conflagraciones extracontinentales que pudieran áfectario o repercutir en la región. El conflicto del Atlántico Sur vino a demostrar que las hipótesis de dificultad podían ser mucho más cercanas y variadas, afectando no solamente su política exterior sino que también otros intereses especificos. Entre ellos puede-señalarse el de las exportaciones brasileñas por la ruta del Atlántico Sur o el de las restricciones en materia de financiamiento internäcional que han afectado a varios países de la región como consecuencia de este conflicto.

Estas y otras dificultades han llevado ya a sugerir los consiguientes replanteamientos en materia de orientación diplomática y militar. En lo primero, se visualiza un papel más activo del Brasil en cuanto a las políticas destinadas a asegurar la paz y la estabilidad regional, lo que pudiera significar la necesidad de abandonar la actitud de prescindencia que ha seguido hasta ahora. En esa misma medida, se vería inevitablemente comprometido en las variaciones de la política regional, como le ha ocurrido a los Estados Unidos. En lo militar ya se escuchan los llamados a una readecuación de los armamentos y estrategias de defensa.

Un segundo ejemplo relevante puede encontrarse en el caso de Chile. El precedente de inestabilidad creado por el conflicto del Atlántico Sur hará que para este país sea imperioso contar con un sistema adecuado de resguardo juridico y de seguridad política, incluyendo mecanismos eficaces para la solución pacífica de controversias y para la prevención de la agresión. En su defecto, existiría la tentación de recurrir a medidas de autodefensa lo que significaría una carrera armamentista muy superior a la que ha habido durante los últimos años. El aislamiento polf́tico de Chile y la presión de algunos sectores interesados ha llevado ya a sugerir tesis en el sentido de que Chile debiera desarrollar una polftica prusiana 
o constituirse en un tipo de Israel latinoamericano. Todo ello no haría sino agravar las condiciones económicas y acentuar la inestabilidad política de la región.

Los ejemplos que se han expuesto no son exclusivos de los países señalados, pues el mismo fenómeno habrá de repetirse respecto de varios otros en función de sus propios problemas. En este sentido, cabría pensar que todos los conflictos latentes o potenciales serán revisados por sus protagonistas a la luz de las nuevas condiciones politicas imperantes en la región, entre los que cabe mencionar destacadamente los de Chile y Argentina, Perú y Ecuador, Colombia y Venezuela.

Todavia es necesario mencionar otra dimensión agravante en el ámbito regional. El conflicto del Atlántico Sur se traducirá ciertamente en una decisión argentina de acelerar el desarrollo de su programa nuclear, en lo cual viene trabajando desde hace más de treinta años en forma ininterrumpida, cualquiera que haya sido el gobierno en el poder. En este plano no cabe descartar que el objetivo último de este programa sea la producción de artefactos nucleares, o al menos contar con la plena capacidad tecnológica para ello. Las reiteradas declaraciones de que este programa sólo persigue fines pacíficos, no excluyen esa posibilidad, pues Argentina entiende que elio permite las explosiones nucleares pacíficas y, en todo caso, se ha negado a suscribir el Tratado de No Proliferación o el de Tlatelolco.

La disponibilidad nuclear argentina no sólo se traducirá en una profunda alteración de las relaciones políticas regionales, sino que además en una carrera armamentista nuclear regional, en la cual cabría desde luego contar a Brasil y a Chile. El grado de peligrosidad que entonces adquiriría el sistema llegaría a ser sumamente grave.

Debe tenerse presente, sin embargo, que no necesariamente las reorientaciones que tengan lugar implicarán en todos los casos o circunstancias una acentuación de los factores negativos propios del elemento de confrontación. También es posible que, afirmándose en la segunda vertiente en que se manifiesta la dicotomía latinoamericana, surjan renovados propósitos de cooperación, particularmente en el plano económico. EI reingreso de varios países al ámbito regional se originó en las dificultades económicas encontradas en su política global, lo que ha determinado un nuevo interés en estas modalidades de cooperación.

La reactivación del sela y de las negociaciones con el Grupo Andino que ha impulsado Argentina, representan los primeros síntomas de esta tendencia, que quizás pueda generalizarse en Sudamérica. Una conclusión pacífica del conflicto bien podría traducinse también en nuevos estímulos en este plano, al igual que las nuevas. 
influencias que puedan hacerse presente en la política interna argentina. En todo caso, ello dependerá fundamentaimente del clima político que prevalezca en la región en los próximos años. Esta alternativa de cooperación sería ciertamente la más deseable. Las iniciativas destinadas a convocar reuniones de cancilleres y presidentes, para tratar de la cooperación regional, responden a esta línea de pensamiento e inquietud.

\section{El deterioro del marco hemisférico}

El tercer plano en que cabe analizar las consecuencias del conflicto es el de las relaciones entre América Latina y los Estados Unidos y la marcha de los organismos interamericanos. En este campo, también las tendencias históricas y los planteamientos contemporáneos que se han examinado tienen una especial relevancia. Dos fueron las etapas asociadas al conflicto que cabe tener presente. La primera consistió en las gestiones de buenos oficios emprendidas por el Secretario de Estado para encontrar una solución pacífica, en tanto que la segunda se inicia al fracasar esos esfuerzos:

La primera etapa fue naturalmente muy bien recibida en América Latina y en la propia Argentina, interpretándose como un nuevo gesto de acercamiento de parte de la administración Reagan, dentro de la línea de conducta que ya hubo ocasión de explicar.

Sin embargo, la seguridad que esas gestiones ófrecfan, parece haber influido en la actitud de intransigencia que en definitiva adoptó el gobierno de Buenos Aires y que fue el factćr precipitante de los muchos acontecimientos que siguieron Una vez que el gobierno de Washington comprobó la imposibilidad de alcanzar resultados satisfactorios, como después habría de comprobarlo el Presidente del Perú y el Secretario General de las Naciones Unidas, tomó la firme decisión de aplicar algunas medidas en contra de Argentina, y, sobre todo, de manifestar su apoyo al Reino Unído.

Esta segunda fase fue la que se tradujo en ciertas expresiones de sorpresa en el ámbito latinoamericano y la que, de acuerdo a algunas opiniones, habria precipitado las relaciones interamericanas en una grave crisis. Sin embargo, esta reacción es la que debe analizarse con especial cuidado, con el fin de apreciar desapasionadamente su alcance y magnitud.

En primer término, cabe señalar que es comprensible el planteamiento de críticas a los Estados Unidos por su actitud, pues ello coincidió precisamente con el momento en que ambas partes intentaban o estudiaban formas de acercamiento. En este sentido, la mezcla de sorpresa y frustración habría de traducirse en las referidas críticas. Pero también debe agregarse que el gobierno norteamericano tuvo escasas opciones disponibles. Ya la opinión públi- 
ca estaba extrañada de que la administración Reagan hubiese adoptado una actitud neutral y discreta en este caso, preguntándose cómo podía tratar en los mismos términos a su más tradicional, fiel y democrático aliado europeo y a un gobierno militar latinoamericano que no reunía ninguna de esas tres características. La presión de la opinión pública, de los medios parlamentarios y de la prensa amenazaba con transformarse en una verdadera crisis política para Reagan, que habría tenido quizás graves consecuencias electorales en el futuro.

Si la solución al conflicto hubiera podido alcanzarse en forma rápida, la actitud equilibrada que inicialmente buscó el Departamento de Estado hubiese podido mantenerse. Desde el momento en que ello no ocurrió así, los Estados Unidos tuvieron que escoger entre difíciles opciones. Con todo, la política norteamericana siguió un curso en cierto modo equíroco, que en definitiva produjo descontento en las dos partes. Mientras Argentina se sentía traicionada por su nuevo y reciente aliado, Gran Bretaña temía una actitud doble de parte del gobierno de Washington. Las discrepancias entre la embajadora Jean Kirkpatrick y el Secretario de Estado acerca de esta materia, así como el confuso incidente del veto y posterior instrucción de abstención en la votación del Consejo de Seguridad sobre un cese del fuego, revelaban la continuada incertidumbre y falta de claridad en la política que se deseaba seguir.

$\mathrm{La}$ necèsidad de respaldar la vigencia del Derecho Internacional y de la solución pacífica de controversias, por una parte, y el deseo de no enemistarse con dos países cuya amistad valorizaba, por otra parte, explican en alguna medida la política oscilante que se siguió en este caso. Además, el primer elemento resultaba también fundamental para dar seguridades a los aliados europeos y a varios países latinoamericanos en el sentido de que Estados Unidos no podfa condonar el uso de la fuerza, pues de Io contrario podía haberse provocado una crisis de confianza todavía mayor, que habría sido particularmente grave para la estabilidad de América Latina.

La reacción adversa que se suscitó en América Latina respecto de la política que siguió el gobierno norteamericano, ha tenido en realidad varias modalidades diferentes. Argentina, como el país más directamente afectado, lanzó una campaña diplomática antinorteamericana, que no sería enteramente exitosa, como lo reveló el viaje del canciller argentino a $\mathrm{La}$ Habana. A pesar de ello no interrumpió enteramente el diálogo con la Casa Blanca. Bajo el actual gobierno de transición es también posible esperar una građual movilización en este plano. Perú y Venezuela, como una nueva expresión de su entendimiento con Buenos Aires, también condenaron fuertemente la política de Ios Estados Unidos, que llegó a calificarse de antihistórica. 
En otros paises, en cambio, la reacción adversa fue más silenciosa y tuvo motivos diferentes. Ella respondía a una inquietud por la situación que pudiera producirse en el campo de la seguridad hemisférica y la manera cómo esto podría afectar las controversias limítrofes y otros problemas regionales, incluyendo en algunos casos el temor de que la Unión Soviética aprovechase estas circunstancias para sus propios fines. Este otro tipo de reacción veía con preocupación los efectos que pudiera tener la política norteamericana en el más largo plazo y no tanto en función de las alternativas del conflicto del Atlántico Sur. Las advertencias que hizo en Washington el Presidente Figueiredo del Brasil se relacionaban más bien con este problema futuro.

Estas diferentes perspectivas explican las varias opiniones que se han manifestado acerca del efecto que todo esto tendrá,en el futuro de las relaciones interamericanas. Como se ha dicho, ellas van desde quienes sostienen que no habrá consecuencia alguna -argumentando de que América Latina siempre ha culpado a Estados Unidos de lo que sucede en la región-, hasta quienes creen que se ha producido una ruptura seria. La realidad pareciera, sin embargo, encontrarse en algún punto intermedio. No pareciera caber dudas de que habrá consecuencias, pero tampoco ellas serán absolutas.

Un primer orden de consecuencias que ya pueden percibirse es el enfriamiento de las relaciones con algunos países de la región. Argentina, Perú y Venezuela representan algunos ejemplos de esta situación. Pero también cabe recordar que en este plano en realidad lo que ha sucedido es que se ha regresado a la tradicional frialdad que había alcanzado el proceso a lo largo de su evolución. Si Ios Estados Unidos pasan a desarrollar una política latinoamericana coherente y una acción diplomática ágil en la región, estas consecuencias podrían minimizarse de manera importante, o en todo caso evitar que se prolonguen por un largo tiempo.

Hay también otras consecuencias que tendrán mayor importancia. La más significativa de ellas se relaciona con la crisis del Sistema Interamericano y de sus organismos. Con anterioridad se señaló que ya el Sistema Interamericano venía reflejando la crisis histórica de las relaciones hemisféricas. Este fenómeno se ha acentuado con motivo del conflicto del Atlántico Sur, hasta tal punto de que ha afectado gravemente la eficacia de esos mecanismos y la del propio Tratado Interamericano de Asistencia Recíproca. La Vigésima Reunión de Consulta fue una importante demostración de las dificultades que enfrenta el sistema, no sólo en cuanto a las diferencias políticas que dividen a los países miembros, sino que también en lo que respecta a las muy diferentes opiniones jurídicas 
sobre las condiciones que podrían hacer procedente la aplicación de ese instrumento.

Las actitudes de desconfianza en la eficacia de la seguridad hemisférica ya se venían manifestando desde que se suscribió el Protocolo de Reformas a ese tratado en 1975 y la discusión sobre el Ievantamiento de las sanciones a Cuba. En ese período, que coincidía con uno de los puntos más bajos en las relaciones hemisféricas, ya se escucharon planteamientos en el sentido de que América Latina debía buscar sus propios arreglos de seguridad, con prescindencia de los Estados Unidos. Hoy día estos planteamientos han surgido nuevamente. Declaraciones del Presidente Herrera Campins, de Venezuela, en el sentido de reestructurar la seguridad regional, o el apoyo dado por Argentina a la antigua aspiración de Costa Rica de constituirse en sede de la OEA, son expresiones de esta tendencia.

El problema de fondo que se plantea en este campo es que el vacío que se ha creado en materia de seguridad hemisférica, desde el momento en que nadie confía en la eficacia de sus instrumentos, puede conducir a una grave inestabilidad en América Latina. De no contarse con instrumentos de esta naturaleza y otros procedimientos expeditos para la solución pacífica de controversias, la aceleración de la carrera armamentista y la búsqueda de alternativas diferentes puede precipitar a América Latina a un tipo de relaciones sumamente conflictiva.

Un riesgo asociado es que en ausencia de mecanismos colectivos, algunos países tengan que asumir el papel de gendarmes de la paz en la región, lo que complicaría todavía más las cosas y se prestaría para todo tipo de pugnas de poder. Ciertamente Estados Unidos no desea ejercer esta función, pues ya tuvo adversas experiencias históricas. Tampoco Brasil desea verse expuesto a tal necesidad, pero, como se vio, Ios efectos del actual conflicto pueden exigirle eventualmente una participación más activa en la política de la región.

En las actuales condiciones es poco probable que llegue a estructurarse un Sistema Latinoamericano de Seguridad, pues ello supondría un intenso proceso de cooperación política y económica y un grado de entendimiento entre los países de la región, que todavía tardará un largo tiempo en madurar. Sin excluir la validez de la tendencia, su factibilidad sólo podrfa apreciarse después de que se dé un conjunto de condiciones que aún no se materializan. Por esta razón, los organismos interamericanos continuarán existiendo, pero requerirán de una profunda reforma, reestructuración y adaptación si se quiere evitar el vacfo a que se ha aludido $y$ sus peligrosas consecuencias. En este sentido, es en el Sistema Inter- 
americano donde habrán de repercutir más intensamente las consecuencias del conflicto del Atlántico Sur.

\section{Dificultades de largo plazo en la escena internacional}

En estrecha relación con la situación de la seguridad continental, cabe todavía mencionar otro aspecto de importancia. En un momento determinado se temió que el conflicto del Atlántico Sur pudiese ser aprovechado por la Unión Soviética para perseguir sus propias finalidades en el Hemisferio Occidental. El óptimo nivel de las relaciones que existían con Argentina, así como sus propias necesidades en materia de abastecimientos de granos y cereales, servían de base para esta inquietud. EI gobierno de Buenos Aires se mostró en un comienzo particularmente interesado en cultivar esta imagen, pues ella podía ser útil como herramienta de presión diplomática. Sucesivas versiones de prensa fueron elocuentes en este sentido, así como declaraciones de las más altas autoridades políticas y militares. El gobierno norteamericano también exteriorizó su preocupación por este aspecto, mediante una advertencia pública del Presidente Reagan de que la unss debía abstenerse de intervenir, como también lo hizo el gobierno británico.

Sin embargo, aparentemente la uRss no tenía el propósito de intervenir en el conflicto mismo, por varias razones. En primer lugar, las relaciones con Buenos Aires no significaban que se hubieran superado las profundas divergencias ideológicas que, separaban a los dos gobiernos, sobre todo con la línea marcadamente anticomunista y prooccidental que había definido originalmente el Presidente Galtieri. Su interés en materia de granos y cereales se vio compensado por el inicio de conversaciones con el gobierno de Estados Unidos en ese campo, que además ofrecía condiciones de pago más favorables que las de Argentina. La conveniencia militar de intervenir en algún sentido tampoco resultaba clara. Igualmente se ha señalado que un apoyo a la causa argentina podía tener resultados contraproducentes en la cuestión de las islas japonesas ocupadas por la Unión Soviética. La abstención de la uRss en el Consejo de Seguridad de las Naciones Unidas, como declaraciones relativas a que observaría una actitud de neutralidad en el conflicto, representan manifestaciones diplomáticas de esa línea de conducta.

Sin que quepa excluir enteramente un interés soviético en brindar algún tipo de ayuda militar -como podría ser el abastecimiento de armamentos-, pareciera ser que las expectativas de ese país se habrían orientado más en función de los efectos futuros del conflicto gue de sus alternativas coyunturales. En particular, el interés podría consistir en capitalizar las reacciones antinorteamerica- 
nas que puedan suscitarse o la inestabilidad que pueda sobrevenir como consecuencia de la crisis de seguridad que se indicó, para así fortalecer su papel en América Latina.

Los gobiernos de Cuba y Nicaragua, que habían mantenido una relación muy hostil con el de Buenos Aires, captaron bien esta expectativa, cambiando de inmediato su posición. El ofrecimiento de apoyo total a la Argentina y el restablecimiento de los respectivos embajadores fue un proceso rápido. El interés argentino de involucrarse en los asuntos centroamericanos desapareció, por cierto, completamente. Todo ello habría de culminar con la visita del Canciller argentino a La Habana con motivo de una reunión del movimiento de los No Alineados, que sirvió de marco apropiado para este estrechamiento de relaciones.

Sin embargo, este último hecho también marcó los límites de la posible apertura argentina hacia el bloque socialista, pues despertó una viva resistencia en la opinión pública y las fuerzas armadas de ese país. Desde Iuego, el Movimiento de los No Alineados no goza de gran prestigio en los medios políticos argentinos, hasta el punto de que el propio Canciller Costa Méndez se había referido a él en términos muy críticos antes de asumir sus funciones de gobierno. En seguida, el hecho de que se calificara a Guba como el orgullo del Caribe, fue interpretado como una concesión excesiva que contradecía las premisas ideológicas básicas del régimen argentino. Más serio aún fue el hecho de que la diplomacia argentinà se uniera a las condenas de esa reunión a Sudáfrica e Israel, países con los que mantiene óptimas relaciones. En el caso de este último, debe recordarse que mantuvo el abastecimiento de armamentos a la Argentina, a pesar del conflicto y de las presiones que recibió para suspenderlo. Además debe tenerse presente el hecho de que Buenos Aires cuenta con una enorme población de origen judío. Por último, el resultado de todo lo anterior fue una magra resoIución que se abstuvo de condenar a Gran Bretaña, dada la influencia de países miembros de la Comunidad Británica de Naciones en ese movimiento.

De esta manera, puede apreciarse que la expectativa de un aprovechamiento soviético es limitada, aún en el largo plazo. Ello no significa que esta eventualidad haya desaparecido, sobre todo si las alternativas de la política hemisférica condujeran a un aumento del conflicto y la inestabilidad en la región.

\section{Las lecciones de un conflicto}

El conflicto del Atlántico Sur ha dejado algunas lecciones amargas para América Latina y para los Estados Unidos. Para la primera, ha indicado claramente los extremos a que puede conducir el na- 
cionalismo exagerado y el aniquilamiento de la democracia, así como el alto precio que debe pagarse por haber abandonado los esfuerzos de cooperación que en una época caracterizaron su actuación regional, habiéndolos reemplazado por esquemas de conflicto y confrontación. Si bien no puede hoy pretenderse que los países latinoamericanos renuncien a las orientaciones internacionales $y$ proyección global que pudieron adquirir durante dos décadas, elemento que continuará presente, ello deberá hacerse sin descuidar el escenario regional que le sirve de contexto y ámbito prioritario de acción.

Si la tendencia a revalorizar el ámbito regional que se ha anotado, pudiera hacerse efectiva mediante el replanteamiento de la cooperación regional, ello sería un resultado altamente positivo de las consecuencias y alternativas que ha dejado ese conflicto. La superación de las divisiones internas en América Latina es la clave para llegar a esta etapa superior de las relaciones entre los países de la región. Con todo lo difícil que ello pueda parecer, no es una tarea imposible. El surgimiento de un nuevo proyecto político latinoamericano, fundamentado en un nuevo liderazgo, constituiría la respuesta adecuada a la actual crisis política y económica que azota a muchos países de esta región. La ideología de la paz y de la cooperación regional quizás emane naturalmente cuando se compruebe el absurdo a que han conducido largos años de violencia y nacionalismo conflictivo.

Para los Estados Unidos, las lecciones no han sido menos amargas. El ideologismo y la valorización del poder con que concibió su política exterior y latinoamericana, han tenido una fuerte influencia en la crisis que hoy afecta a las relaciones hemisféricas. Ello no sólo se tradujo en políticas imprecisas y poco definidas, sino que también en signos equívocos de lo que Estados Unidos deseaba de sus relaciones con América Latina.

Quizás la situación que se ha creado contribuiría a replantear esa política, lo que también representaría una positiva reacción. Dos parecieran ser las orientaciones que se imponen en este sentido. En primer lugar, el gobierno norteamericano debería hacer explícita su ideología democrática como componente de su política exterior, campo en el cual no puede permitirse que se entiendan las señales equivocadas. Ello contribuiría a otorgarle una importante claridad a esa política, estableciendo los límites que las ideologías del nacionalismo, del marxismo o cualquiera otra no podrán sobrepasar. La ausencia de este criterio explícito estuvo estrechamente vinculada al desencadenamiento de la actual crisis en el Atlántico Sur.

La segunda orientación que se impone es la necesidad de estimular la cooperación regional mediante la participación activa de todos los países de la región. La selectividad asociada a la política 
del poder que se venía siguiendo, impidió que estas intenciones pudieran materializarse. El signo en cierto modo negativo que caracterizaba esa política, debe reemplazarse por otro de carácter positivo. En este marco tendría especial influencia la reiteración de algunos principios positivos de la política internacional, como la necesidad de conducir las relaciones internacionales en conformidad al Derecho Internacional y la obligación de solucionar las controversias mediante los procedimientos pacíficos que éste prevé. También ello contribuiría a evitar equívocos innecesarios y a dotar esa política de un claro contenido.

Si tanto América Latina como los Estados Unidos extraen las conclusiones apropiadas del conflicto en cuestión, es quizá posible que las relaciones hemisféricas adquieran una nueva vitalidad. Se alcanzaría así la etapa de convergencia que resultó paralizada como consecuencia de la incertidumbre reciente de la política norteamericana y de la situación del Atlántico Sur. Si América Latina se había mostrado interesada en ellos, nada impediría que lo siga estando en el marco de un nuevo proyecto político hemisférico, que respete su identidad y autonomía y facilite los objetivos de la cooperación. También los Estados Unidos parecían interesados en esta nueva perspectiva, Ia que podría alcanzarse mediante las redefiniciones señaladas y una diplomacia refinada y activa.

Los intereses respectivos parecieran justificar una convergencia de políticas. Ninguna de las partes dejaría de mirar a la consecución de sus propios objetivos regionales y globales, que ya forman parte de lo adquirido a través de la evolución de sus relaciones internacionales, pero podría valorizar el aporte que la otra pueda efectuar. El logro de un nuevo entendimiento significaría que América Latina y los Estados Unidos han alcanzado una etapa madura de su vinculación internacional, llegando a una fase positiva del ciclo histórico tan complejo y prolongado que ha tenido lugar. 